\title{
Surface resistivity measurements and related performance studies of the Bakelite RPC detectors
}

\author{
K. K. Meghna ${ }^{1,2}$, A. Banerjee ${ }^{3}$, S. Biswas ${ }^{* 3,4}$, S. Bhattacharya ${ }^{2}$, S. Bose ${ }^{2}$, \\ S. Chattopadhyay ${ }^{3}$, G. Das ${ }^{3}$, C.Marick ${ }^{2}$, S. Saha ${ }^{2}$, Y.P. Viyogi ${ }^{3}$ \\ ${ }^{1}$ Institute of Mathematical Sciences, Chennai-600 113, India \\ ${ }^{2}$ Saha Institute of Nuclear Physics, 1/AF Bidhan Nagar, Kolkata-700 064, India \\ ${ }^{3}$ Variable Energy Cyclotron Centre, 1/AF Bidhan Nagar, Kolkata-700 064, India \\ ${ }^{4}$ GSI Helmholtzzentrum für Schwerionenforschung GmbH, Darmstadt, Germany \\ ${ }^{*}$ E-mail: saikat.ino@gmail.com
}

\begin{abstract}
Bakelite RPC detectors of various sizes from $10 \mathrm{~cm} \times 10 \mathrm{~cm}$ to $1 \mathrm{~m} \times 1 \mathrm{~m}$ have been fabricated, characterized and optimized for efficiency and time resolution. Thin layers of different grades of silicone compound are applied to the inner electrode surfaces to make them smooth and also to reduce the surface resistivity. Effectiveness of different silicone coating in modifying the surface resistivity was evaluated by an instrument developed for monitoring the I-V curve of a high resistive surface. The results indicate definite correlation of the detector efficiency for the atmospheric muons and the RPC noise rates with the surface resistivity and its variation with the applied bias voltage. It was also found that the surface resistivity varies for different grades of silicone material applied as coating, and the results are found to be consistent with the detector efficiency and noise rate measurements done with these RPCs.
\end{abstract}

XI Workshop on Resistive Plate Chambers and Related Detectors

5-10 February, 2012

Laboratori Nazionali di Frascati dell'INFN - Frascati (Rome) - Italy

\footnotetext{
* Speaker.
} 


\section{Introduction}

The Resistive Plate Chamber (RPC) detectors, first developed by Santonico et al. [1] are being used extensively in high energy physics and neutrino physics experiments. The large area RPC modules are used in experiments like BELLE, BaBar, BESIII, and several LHC experiments (ALICE, ATLAS, CMS etc.) mainly for a) relatively low cost of materials used in making RPCs, b) robust fabrication procedure and handling and c) good time and position resolutions. RPCs are used in neutrino experiments like OPERA where its excellent time resolution and tracking capabilities are exploited.

During the last few years significant work on the prototype silicone coated bakelite based RPC for the Iron Calorimeter (ICAL) of the proposed India-based Neutrino Observatory (INO) has been carried out at SINP/VECC [2]. INO is being planned to determine the neutrino oscillation parameters precisely in the 3-flavor mixing scenario using atmospheric neutrinos. For effective separation of up-coming and down-going neutrinos and background rejection, ICAL requires highly efficient and sensitive detectors with $\lesssim 2$ ns time resolution. Bakelite RPC detectors of various sizes from $10 \mathrm{~cm} \times 10 \mathrm{~cm}$ to $1 \mathrm{~m} \times 1 \mathrm{~m}$ have been fabricated, characterized and optimized for efficiency and time resolution, and are reported earlier [3, 4, 5, 6, 7, 8]. For RPC electrodes, careful choice of materials, smoothness of surfaces to avoid localization of excess charges, surface treatment to reduce the surface resistivity or providing alternate leakage path for post-streamer recovery are adopted in the major high energy physics experiments. The current paper deals with the development of instruments and methods for the measurement of surface resistivity of the inner electrodes of the baklite RPCs. Incidentally, these developments are complimentary to the development of glass-based RPCs in other collaborating institutes of the proposed INO project.

\section{Experimental set-up}

Thin layers of different grades of silicone compounds are applied to the inner electrode surfaces to make them smooth and also to reduce the surface resistivity. Before fabrication of each RPC module, the surface resistivity of bakelite sheets and also of silicone coated surfaces are measured using the set-up shown schematically in the Figure 1. The actual experimental set-up is shown in the Figure 2. The set-up consists of a jig with two aluminium bars having V-shaped sections (shown in the inset to Figure 2) and soft-padded conducting edges at the bottom, which are placed on the surface under measurement. The bars, forming the opposite sides of a square shape, were mounted on a G-10 insulating plate having very high resistivity $\left(>10^{14} \Omega / \square\right)$. The length of the aluminium bars and their separation were same $(5 \mathrm{~cm})$. A current to voltage converter circuit, made out of TL082CN FET input OPAMP, with provisions to cover 3 decades of surface resistivity measurement $\left(\sim 10^{10}-10^{12} \Omega / \square\right)$, was made.

Measurements were done on the innner surfaces of the bakelite electrodes (silicone coated or uncoated) before assembly of the RPCs. A DC bias voltage $\sim 50-600$ volt was applied on the jig, and the leakage current $(\sim \mathrm{nA} / \mathrm{pA})$ flowing across the terminals of the jig through the bakelite surface was measured. The surface resistivity was obtained from the leakage current and the applied bias voltage. 


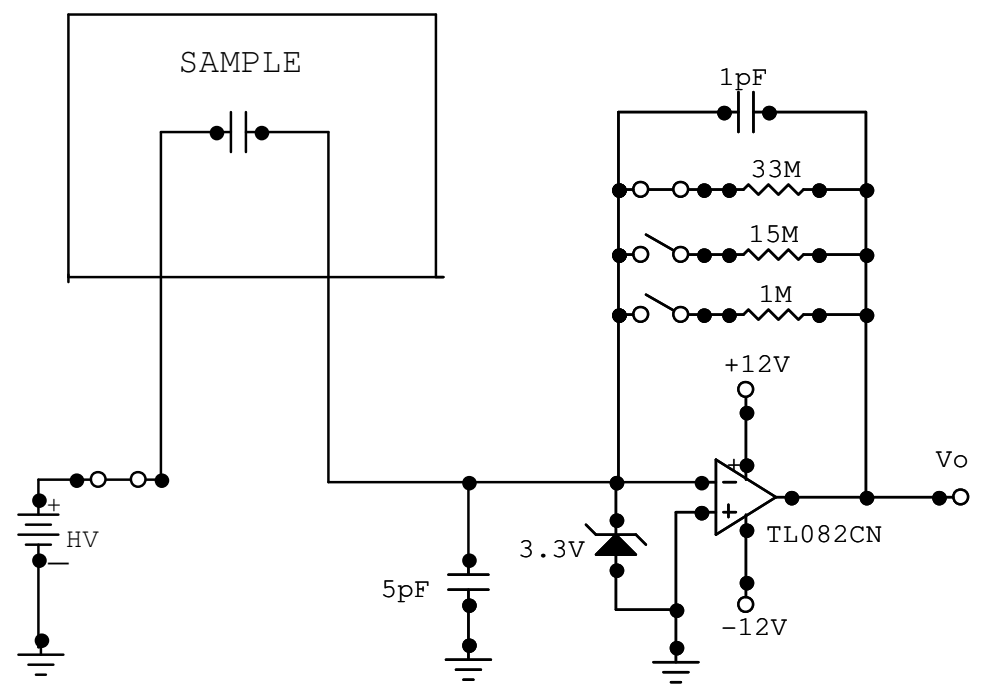

Figure 1: Block diagram of the surface resistivity measurement setup.

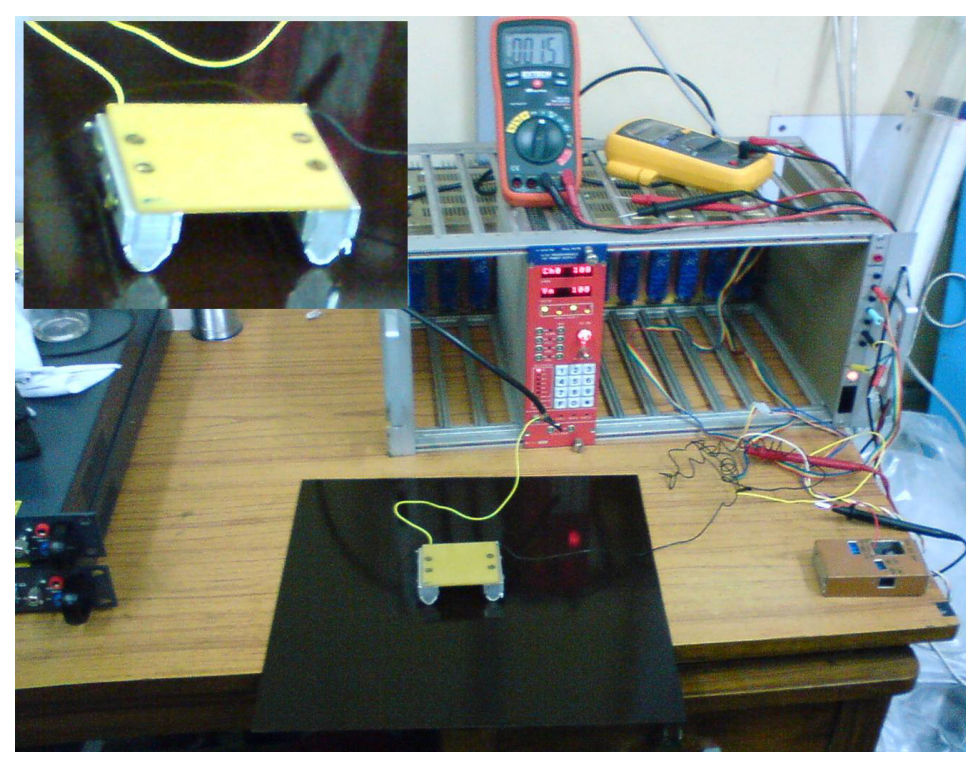

Figure 2: Experimental setup for surface resistivity measurement. The inset shows the aluminium jig used for the purpose.

The bakelite sheets used for making the RPC electrodes were obtained in two batches, having different surface textures. The samples from the bakelite sheets were scanned under an Atomic Force Microscope (AFM) to determine their roughness. The AFM plots for the two samples (rough (a) and smooth (b)) are shown in the Figure 3 side by side for comparison. The average roughness for the 'rough' and the 'smooth' surfaces were found to be $100 \mathrm{~nm}$ and $18 \mathrm{~nm}$ respectively. The surface resistivity was measured for these two surface grades, along with the coatings applied on them. The results are discussed in the following section. 

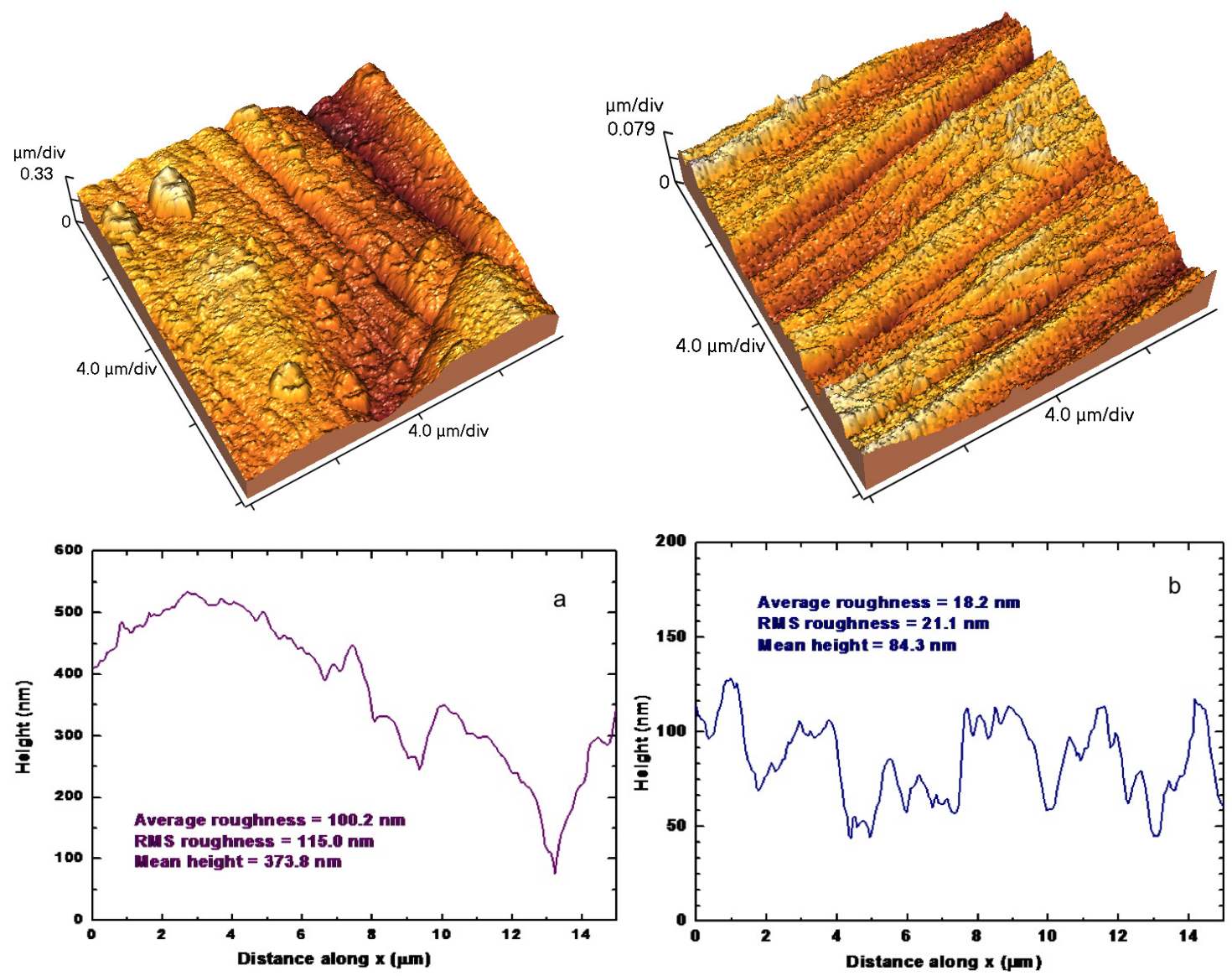

Figure 3: AFM image and topography of AFM data for the (a) rough and (b) smooth bakelite surface morphological structure.

\section{Results}

The variation of surface current and that of surface resistivity with the applied voltage for different coated and uncoated bakelite electrodes are shown in Figure 4 (a) and 4 (b) respectively. A look at the I-V plots for the surface resistivity of uncoated bakelites having two different surface textures (rough and smooth) shows a non-linear trend for the rough surface, resulting in an apparent reduction of surface resistivity at higher bias voltage. This is likely to be correlated with the relatively high occurence of micro-discharge across the surface due to the roughness. This finding correlates with the reduction of efficiency and increase of RPC noise rate at higher bias voltages for the bakelite RPCs, made with the rough variety in our earlier studies [4]. In addition, comparison of the two different grades of silicone coating (unpolymerized and polymerized) on the bakelite electrode surface shows that 1 ) the surface resistivity is less by a factor of $\sim 2$ for the coated surface compared to the uncoated surface, and 2) the surface resistivity for the polymerized silicone coating is $\sim 1.5$ times higher than that for the unpolymerized variety. Lower value of surface resistivity is expected to help in reducing the space charge effect because of quicker dissipation of accumulated charge through the surface layer. 

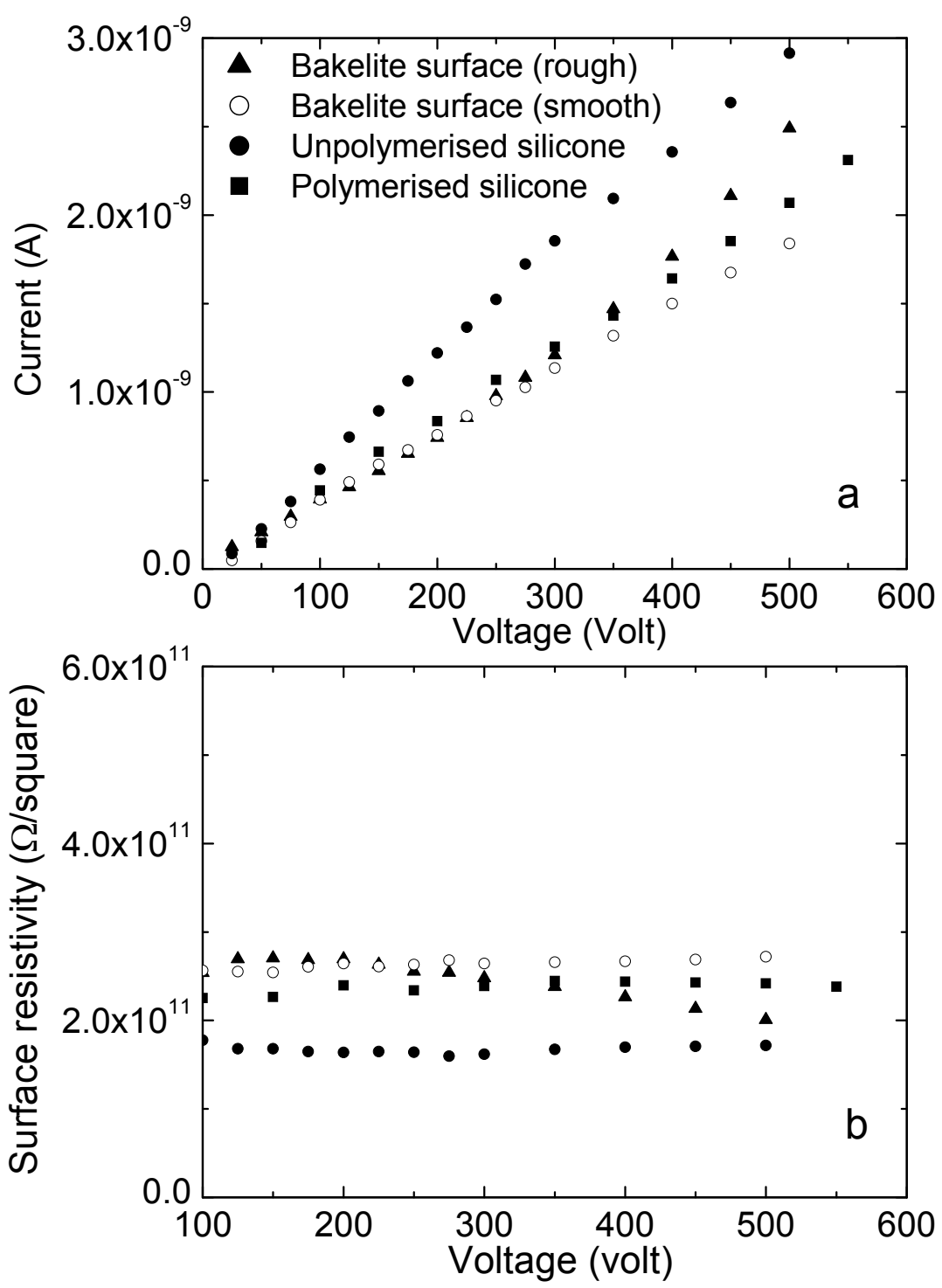

Figure 4: (a) The surface current \& (b) the surface resistivity versus the applied voltage for different samples.

It may be noted that RPCs made by silicone coated (unpolymerised) bakelite plates as electrodes have been tested earlier for a long duration showing $\sim 96 \%$ efficiency for a period of operation of more than 130 days [4]. The time resolution for the RPCs was found to be $\sim 2$ ns [6] and the measured average charge content per pulse is $\sim 100 \mathrm{pC}$ at an applied high voltage of $8 \mathrm{kV}$ in the streamer mode of operation[7]. 


\section{Conclusions and outlook}

In conclusion, an instrument is developed to measure the surface resistivity of high resistive $\left(\sim 10^{10}-10^{12} \Omega / \square\right)$ surface such as glass and bakelite. This measurement is very essential before fabrication of RPC modules. Using this instrument, measurements were done on the innner surfaces of several bakelite electrodes (silicone coated or uncoated) before fabricating the detectors. The surface resistivity of the silicone coated (unpolymerised) surface was found to be less by a factor of 2 compared to the uncoated surface and by a factor of $\sim 1.5$ compared to the polymerised silicone coated variety. These findings correlate well with our earlier observation on the long term stability of performance of the unpolymerized silicone coated bakelite RPC detectors.

\section{References}

[1] R. Santonico, R. Cardarelli, Nucl. Inst. and Meth. 187, (1981) 377.

[2] INO Project Report, INO/2006/01, June 2006, 〈http://www.imsc.res.in/ ino/>.

[3] S. Bose, et al., Nucl. Instr. and Meth. A 602 (2009) 839.

[4] S. Biswas, et al., Nucl. Instr. and Meth. A 602 (2009) 749.

[5] S. Biswas, et al., Nucl. Instr. and Meth. A 604 (2009) 310.

[6] S. Biswas, et al., Nucl. Instr. and Meth. A 617 (2010) 138.

[7] S. Biswas, et al., Nucl. Instr. and Meth. A 661 (2012) S94.

[8] PhD Thesis, Saikat Biswas, 2011, University of Calcutta. 